\title{
ANÁLISIS DE UN SISTEMA SILVOPASTORAL CON PinUS radiata D. DON. ASOCIADO CON GANADO OVINO EN LA ZONA MEDITERRÁNEA COSTERA CENTRAL DE CHILE.
}

\author{
Alvaro Sotomayor Garreton ${ }^{1}$ y Cesar Cabrera Severino ${ }^{2}$
}

\section{RESUMEN}

En el año 1983 se estableció un sistema silvopastoral con Pinus radiata D. Don, el cual se compara en su productividad y rentabilidad con dos sistemas de uso tradicional en el secano costero de la Región de O' Higgins, Chile; el manejo ganadero ovino y el sistema forestal con Pinus radiata.

El sistema silvopastoral fue diseñado a una densidad de 625 árboles por hectárea, plantados en conglomerados de 4 plantas, con un espaciamiento a dos por dos metros entre plantas y a 6 metros entre conglomerados, combinados con tres tipos de pradera (pradera sembrada de trébol subterráneo y falaris, pradera natural mejorada con fertilización y pradera natural sin fertilización), con un manejo animal con ovinos, el cual fue manejado con podas y raleos terminando con una densidad final promedio de 200 arb.ha-1, podado a 7 metros de altura. El sistema forestal puro se inició con una densidad de 1.600 arb.ha ${ }^{-1}$, llegando al final de la rotación con 500 arb.ha' ${ }^{-1}$ podado a 4.1 metros de altura. El manejo del sistema ganadero con ovinos, con las mismas praderas utilizadas en el sistema silvopastoral, fue el tradicional usado por los productores de dicha zona.

Los resultados del estudio arrojan una rentabilidad similar entre los sistemas silvopastorales con pradera natural mejorada y pradera natural, demostrando que los sistemas silvopastorales entregan una interesante alternativa para los productores del secano costero de la Región de O'Higgins, dado que les permite obtener ingresos intermedios por la venta de carne y lana, y madera proveniente de raleos, mientras que obtienen un ingreso final por la venta de madera a los 24 años de rotación por el componente forestal. Los sistemas ganaderos fueron los que obtuvieron la menor rentabilidad entre los sistemas comparados, siendo en todos los casos el de menor rentabilidad cuando se utiliza la pradera sembrada, tanto en sistema silvopastorales como pastorales puro, por el alto costo de la fertilización anual que este tipo de pradera requiere.

\footnotetext{
' Ingeniero Forestal, M Sc., Investigador Instituto de Investigacion Forestal de Chile (INFOR), Sede Bio Bio, Concepcion, Chile; asotomay@infor.cl.

${ }^{2}$ Ingeniero Forestal, Corporación Nacional Forestal (CONAF), Rancagua, Chile; ccabrera@conaf.cl
} 


\section{ANALYSIS OF A SILVOPASTORAL SYSTEM WITH Pinus radiata D. DON, ASSOCIATED WITH OVINES IN THE CENTRAL COASTAL MEDITERRANEAN ZONE OF CHILE.}

\section{SUMMARY}

In 1983 a silvopastoral system with Pinus radiata D Don was established, which is compared in its productivity and yield with two systems of traditional use in the coastal dry land of Region of O'Higgins, Chile, as they are the ovine cattle handling and a forest system with Pinus radiata.

The silvopastoral system was designed to a density of 625 trees by hectare, planted in conglomerates of 4 plants, spaced two by two meters between plants, and spaced 6 meters between conglomerates, combined with three types of prairie (prairie seeded of subterranean clover and fallaris; natural prairie improved with fertilization; and natural prairie without fertilization), with a handling animal with ovines, which was managed with thinnings and prunings and finishing with a final density 200 average of trees ha ${ }^{-1}$, pruned to 7 meters height. The forest system beginning with a density of 1,600 trees ha ${ }^{-1}$, arriving at the end of the rotation with 500 trees ha-1 pruned at 4.1 meters height. The handling of the cattle system with ovines, with the same prairies used in the silvopastoral system, was the traditional one used by the producers of this zone

The results of the study shows a similar yield between the silvopastoral systems with natural improved prairie and natural prairie, with de forest system, demonstrating that the silvopastoral systems give an interesting alternative for the producers of the coastal dry land of the O'Higgins Region, since it allows them to obtain intermediate income by the sale of meat and wool, and wood from thinnings, whereas they obtain a final entrance by the sale of wood at the end of rotation, 24 years, by the forest component. The traditional cattle systems were those that obtained the smaller yield between the compared systems, being in all the cases the one of smaller yield when the seeded prairie is used, as much in pure silvopastoral as pastoral system. because of the high cost of the annual fertilization that this type of prairie requires. 


\section{INTRODUCCIÓN}

En la actualidad los pequeños propietarios silvoagropecuarios ubicados en el secano costero de la Región de O'Higgins, Chile, destinan gran parte de sus suelos a la actividad ganadera extensiva, principalmente con ovinos, sin resguardo de la capacidad de uso de los suelos, encontrándose vastas extensiones de suelos de capacidad de uso VI y VII (forestal-ganadera), cubiertas con praderas naturales de regular a mala calidad, con una baja productividad para la producción de carne y/o lana y con suelos erosionados producto de un sobretalajeo y de una baja protección con vegetación perenne sobre estos. Lo anterior debido que es uno de los rubros que les permite ingresos anuales, junto con cultivos anuales como trigo, cebada, lentejas y otros, tanto para el autoconsumo, como para la venta.

Por lo anteriormente señalado, el mejoramiento de la rentabilidad de los predios de la Región, o al menos de un importante segmento de ellos, pasa por innovar e incorporar tecnologias adecuadas, que permitan mejorar la eficiencia de producción de sus rubros tradicionales y que considere la sustentabilidad del sistema utilizado. Dentro de esta innovación e incorporación de tecnologia, es posible la integración del rubro forestal y ganadero en forma conjunta como sistema productivo, llamado "Sistema Silvopastoral", dentro de un mismo espacio físico, potrero, o bajo un ordenamiento predial, que permita generar una simbiosis, en la cual se vean beneficiados ambos rubros.

Desde el punto de vista técnico, con la incorporación de árboles en las unidades prediales destinadas a la ganadería tradicional, ordenados de acuerdo a algún diseño silvopastoral, se puede ayudar a incrementar la productividad de los recursos agropecuarios, principalmente en la producción de forraje y producción animal con ganado ovino o bovino, por el mejoramiento de las condiciones ambientales dentro de su área de influencia

La protección brindada por los árboles a los vegetales y animales se expresa en disminución de la velocidad del viento, aumento de la temperatura ambiental, del suelo y de la humedad relativa, disminución del déficit hidrico, protección del ganado contra lluvias y bajas temperaturas, reducción de la erosión por viento o lluvias, y otros aspectos (Sotomayor et al., 2004; Sotomayor, 1989; Sotomayor, 1990; www.agroforesteria.cl; Quam \& Johnson, 1999).

La componente forestal incorporada por su parte se ve beneficiada por el ganado en el sistema (Anderson et al, 1988), por el consumo de malezas por parte de este que evita la competencia con los árboles, en especial en los primeros años de crecimiento (disminuyendo a su vez la posibilidad de incendios forestales); por el reciclaje de nutrientes en el suelo; también estará beneficiada por las fertilizaciones que reciban las praderas y cultivos forrajeros asociados a las plantaciones; y por el mayor espacio dejado para el crecimiento de los árboles, producto del ordenamiento agroforestal.

Desde el punto de vista económico, los productores locales que incorporen estas tecnologias mixtas y las integren dentro de sus predios podrán mejorar su flujo de caja anual por la venta de animales (carne y/o lana), que permitirá solventar los gastos propios del grupo familiar, como también del manejo de los bosques mientras estos maduran y entreguen productos (Sotomayor, 1990) y asi mejorar su calidad de vida (Leslie, et al., 1998; Polla, 1998). Podrán obtener recursos madereros intermedios, como metros ruma, madera para postes 
o leña, al momento de efectuar las labores de raleos y poda y, finalmente, podrán obtener productos maderables de alta calidad (madera libre de nudos o con nudos firmes), al final de la rotación de la plantación forestal al haber manejado sus bosques. Además, se puede señalar que los predios se valorizarán, al momento de establecer una masa forestal dentro de ellos.

Todo este sistema integrado de producción silvopastoral, se caracterizará por su sustentabilidad, ya que permitirá recuperar terrenos degradados, controlar los procesos de erosión, proteger los cursos de agua y mejorar la calidad del agua, aumentar la capacidad de captura del $\mathrm{CO}_{2}$ atmosférico (www.agroforesteria.cl) y, al mismo tiempo, cumplirá con generar un paisaje estéticamente más agradable y mantener un hábitat para la vida silvestre.

\section{OBJETIVOS}

Comparar la productividad de sistemas silvopastorales con Pinus radiata asociados a tres tipos de pradera y ganado ovino, con los sistemas tradicionales de uso del suelo con ganado ovino, y una situación de manejo forestal tradicional, en el secano costero de la Región de O'Higgins, Chile.

\section{ANTECEDENTES GENERALES}

El presente estudio esta siendo desarrollado en el Centro Experimental Forestal (CEF) Tanume, ubicado en la Comuna de Pichilemu, Región de O'Higgins, Chile.

\section{Descripción de la Unidad Experimental, CEF Tanume}

Sector: Tanume, Predio costero, $40 \mathrm{~km}$ al norte de la Ciudad de Pichilemu, Región de O'Higgins.

Comuna: Pichilemu

Provincia: Cardenal Caro

Ubicación Geográfica: $34^{\circ} 15^{\prime}$ LS y $74^{\circ} 49^{\circ}$ LW.

Propietario del predio: CONAF

Especies Forestales Utilizadas: Pinus radiata D. Don

Tipo de Pradera: Pradera sembrada con Trébol subterraneo (Trifolium

subterraneum) y falaris (Phallaris aquatica $c v$ sirosa y $c v$ steptanera), pradera natural mejorada con fertilización, y pradera natural sin fertilización.

Especies Animales Utilizadas: Ovinos, Raza Merino Precoz

\section{Caracterización de la Unidad Experimental}

Esta unidad se ubica en el predio Tanume a unos $40 \mathrm{~km}$ al norte de la ciudad de Pichilemu, ubicada en una zona de clima Templado Subhumedo, sobre una terraza marina, ubicada en el secano costero de la Región. 


\section{Cuadro $\mathrm{N}^{\circ} 1$ \\ PARÁMETROS CLIMÁTICOS SECANO COSTERO REGIÓN DE O'HIGGINS}

\begin{tabular}{|l|c|}
\hline \multicolumn{1}{|c|}{ Parámetros } & CLIMA \\
\cline { 2 - 3 } & Templado Sub Húmedo \\
\hline $\mathrm{T}^{\circ}$ media anual $\left(\mathrm{C}^{\circ}\right)$ & 11,6 \\
\hline $\mathrm{T}^{\circ}$ media minima $\left(\mathrm{C}^{\circ}\right)$ & $\mathbf{8 , 6}$ \\
\hline Periodo libre heladas (meses)) & 9 \\
\hline Precipitaciones (mm año-1) & 705 \\
\hline Período Seco (meses) & 8 \\
\hline
\end{tabular}

\section{MATERIAL Y MÉTODO}

Para evaluar la factibilidad de la combinación silvopastoral en el secano costero de la región y con el fin de entregar un sistema integral de manejo sustentable de los predios de pequeños propietarios y así mejorar su productividad, el estudio consideró la implementación de diferentes módulos experimentales para comparar los usos tradicionales en la zona, como son los usos forestales con Pinus radiata y los usos ganaderos con ovinos, versus un sistema silvopastoral con Pinus radiata asociado a producción ovina y diversos tipos de praderas en terrenos del CEF Tanume, de propiedad de CONAF.

Se instaló durante el año 1983 unidades de investigación de 6 ha cada con el fin de estudiar el comportamiento de la especie Pinus radiata bajo densidades silvopastorales (625 arb.ha') y manejo intensivo con ovinos, y su influencia en la productividad de una pradera naturalizada, mejorada y una sembrada; además se compara con un sistema ganadero con ovinos con las mismas tres tipos de praderas $y$, con un sistema forestal tradicional con una densidad de 1.600 arb.ha $^{-1}$ al establecimiento, y con un manejo forestal apropiado para la pequeña propiedad. 


\section{Tratamientos}

El detalle de los tratamientos instalados, su diseño y superficie, está resumido en el Cuadro $\mathrm{N}^{\circ} 2$.

\section{Cuadro 2}

\section{DESCRIPCIÓN DE TRATAMIENTOS EN CEF TANUME}

\begin{tabular}{|c|c|c|c|c|c|}
\hline \multirow{3}{*}{ Tratamiento } & \multicolumn{3}{|c|}{ Componente arbóreo } & \multirow{3}{*}{ Tipo de Manejo } & \multirow{3}{*}{ Esquema de Plantación } \\
\hline & \multicolumn{2}{|c|}{ Densidad arb.ha' ${ }^{-1}$} & \multirow{2}{*}{$\begin{array}{l}\text { Altura Poda } \\
\text { planificada } \\
\text { (m) }\end{array}$} & & \\
\hline & Inicial & $\begin{array}{c}\text { Final } \\
\text { Esperada }\end{array}$ & & & \\
\hline T 1: PS 625 & 625 & 200 & 7,0 & $\begin{array}{l}\text { Sistema Silvopastoral } \\
\text { con producción ovina, } \\
\text { pradera sembrada de trébol } \\
\text { subterráneo y falaris, asociado } \\
\text { con una cubierta arbórea de } \\
\text { Pinus radiata }\end{array}$ & $\begin{array}{l}\text { Plantación en conglomerados } \\
\text { de } 4 \text { plantas, de Pinus } \\
\text { radiata plantadas a } 2 \times 2 \mathrm{~m} \text {, } \\
\text { espaciadas a } 6 \mathrm{~m} \text { entre ellos, } \\
(2 \times 2) \times 6 \mathrm{~m}\end{array}$ \\
\hline T 2: PM 625 & 625 & 200 & 7,0 & $\begin{array}{l}\text { Sistema Silvopastoral con } \\
\text { producción ovina, pradera } \\
\text { natural mejorada con régimen } \\
\text { de fertilización, asociado con } \\
\text { una cubierta arbórea de Pinus } \\
\text { radiata }\end{array}$ & $\begin{array}{l}\text { Plantación en conglomerados } \\
\text { de } 4 \text { plantas, de Pinus } \\
\text { radiata plantadas a } 2 \times 2 \mathrm{~m} \text {, } \\
\text { espaciadas a } 6 \text { m entre ellos, } \\
\text { espaciamiento a }(2 \times 2) \times 6 \mathrm{~m}\end{array}$ \\
\hline T 3: PN 625 & 625 & 200 & 7,0 & $\begin{array}{l}\text { Sistema Silvopastoral con } \\
\text { producción ovina, pradera } \\
\text { natural, asociado con una } \\
\text { cubierta arbórea de Pinus } \\
\text { radiata }\end{array}$ & $\begin{array}{l}\text { Plantación en conglomerados } \\
\text { de } 4 \text { plantas, de Pinus } \\
\text { radiata plantadas a } 2 \times 2 \mathrm{~m} \text {, } \\
\text { espaciadas a } 6 \mathrm{~m} \text { entre ellos, } \\
\text { espaciamiento a ( } 2 \times 2) \times 6 \mathrm{~m}\end{array}$ \\
\hline T4: Forestal 1600 & 1600 & 500 & 4,10 & $\begin{array}{l}\text { Manejo forestal con podas y } \\
\text { raleos con fines de producción } \\
\text { de madera; sin manejo } \\
\text { ganadero }\end{array}$ & $\begin{array}{l}\text { Plantación a una densidad de } \\
1600 \text { arb/ha, con espaciamiento } \\
\text { a } 2 \times 3 \mathrm{~m}\end{array}$ \\
\hline T5: PS & $\cdot$ & $\cdot$ & $\cdot$ & $\begin{array}{l}\text { Sistema ganadero con } \\
\text { producción ovina, y } \\
\text { pradera sembrada de trébol } \\
\text { subterráneo y falaris. }\end{array}$ & Sin árboles \\
\hline T6: PM & $\cdot$ & - & - & $\begin{array}{l}\text { Sistema ganadero con } \\
\text { producción ovina y pradera } \\
\text { natural mejorada con } \\
\text { fertilización. }\end{array}$ & Sin árboles \\
\hline T7: PN & $\cdot$ & $\cdot$ & & $\begin{array}{l}\text { Sistema ganadero con } \\
\text { producción ovina y pradera } \\
\text { natural sin fertilización. }\end{array}$ & Sin árboles \\
\hline
\end{tabular}

PS: Pradera sembrada; PM: Pradera Mejorada; PN: Pradera Natural 625: densidad 625 arb.ha' ${ }^{-1}$; 1600: densidad 1600 arb.ha ${ }^{-1}$

(PS fue establecida en conjunto con un cultivo de trigo) 


\section{Descripción de los Tratamientos}

-T1: Sistema Silvopastoral (PS 625).

Pinus radiata plantado a 625 arb.ha $^{-1}$ - pradera sembrada - ganado ovino

\section{Cuadro $\mathrm{N}^{\circ} 3$}

ESQUEMA DE MANEJO SISTEMA SILVOPASTORAL DE Pinus radiata CON 625 arb.ha-1 EN CONGLOMERADO CON PRADERA SEMBRADA Y GANADO OVINO

\begin{tabular}{|c|c|c|c|}
\hline Año & $\begin{array}{l}\text { Densidad } \\
\text { residual } \\
\text { (arb.ha-1) }\end{array}$ & $\begin{array}{c}\text { Arboles a } \\
\text { podar o extraer } \\
(\text { arb.ha-1) }\end{array}$ & Actividad \\
\hline $\begin{array}{c}1 \\
3 \\
5 \\
6 \\
7 \\
8 \\
10 \\
10 \\
24 \\
\\
2 \\
2 \\
2,3,4,5 \\
6,7,9,10,11\end{array}$ & $\begin{array}{l}625 \\
625 \\
625 \\
625 \\
318 \\
313 \\
226 \\
226 \\
200\end{array}$ & $\begin{array}{l}468 \\
318 \\
313 \\
226 \\
200 \\
200 \\
200\end{array}$ & $\begin{array}{l}\text { Establecimiento de la plantación: } \\
\text { Habilitación de terreno (roce y ordenamiento desechos) } \\
\text { Preparación del suelo para plantación (subsolado, camellones } \\
\text { curva nivel) } \\
\text { Construcción de cerco, control de malezas preplantación } \\
\text { Plantación densidad } 625 \text { arb. ha' }{ }^{-1} \text {, en conglomerados de } 2 \times 2 \text { m y } 6 \\
\text { m entre conglomerados. } \\
\text { Fertilización con boro y NPK. } \\
\text { Manejo y Mantención de plantación: } \\
\text { Control malezas postplantación } \\
\text { Corrección de forma (eliminar daños por helada y polilla) } \\
\text { Poda } 1 \text { (máximo } 30-40 \% \text { de la altura del árbol) } \\
\text { Raleo } 1 \text { a desecho } \\
\text { Poda } 2 \text { (máximo } 20-25 \% \text { copa viva del àrbol) } \\
\text { Raleo comercial } \\
\text { Poda final (hasta } 7 \text { m) } \\
\text { Raleo comercial } \\
\text { Cosecha Final } \\
\text { Establecimiento pradera } \\
\text { Preparación suelo (aradura, rastraje) } \\
\text { Siembra } \\
\text { Fertilización } \\
\text { Fertilización } \\
\text { Manejo ganadero (ovinos) } \\
\text { Construcción corral } \\
\text { Introducción ganado } \\
\text { Vigilancia ganado } \\
\text { Transporte ganado } \\
\text { Gastos financieros ganado }\end{array}$ \\
\hline
\end{tabular}


T2: Sistema Silvopastoral (PM 625).

Pinus radiata 625 arb.ha* ${ }^{-1}$ - pradera mejorada - ganado ovino.

Cuadro $\mathrm{N}^{\circ} 4$

ESQUEMA DE MANEJO SISTEMA SILVOPASTORAL DE Pinus radiata CON 625 arb.ha-1 EN CONGLOMERADO CON PRADERA MEJORADA Y GANADO OVINO

\begin{tabular}{|c|c|c|c|}
\hline Afío & $\begin{array}{l}\text { Densidad } \\
\text { residual } \\
\text { (arb.ha-1) }\end{array}$ & $\begin{array}{c}\text { Arboles a podar } \\
\text { o extraer } \\
\text { (arb.ha-1) }\end{array}$ & Actividad \\
\hline $\begin{array}{c}1 \\
3 \\
5 \\
6 \\
7 \\
8 \\
10 \\
10 \\
24\end{array}$ & $\begin{array}{l}625 \\
625 \\
625 \\
625 \\
318 \\
313 \\
226 \\
226 \\
200\end{array}$ & $\begin{array}{l}468 \\
318 \\
313 \\
226 \\
200 \\
200 \\
200\end{array}$ & $\begin{array}{l}\text { Establecimiento de la plantación: } \\
\text { Habilitación de terreno (roce y ordenamiento desechos) } \\
\text { Preparación del suelo para plantación (subsolado, } \\
\text { camellones curva nivel) } \\
\text { Construcción de cerco, control de malezas preplantación } \\
\text { Plantación densidad } 625 \text { arb. ha-1, en conglomerados de } 2 \times 2 \\
\text { m y } 6 \text { m entre conglomerados. } \\
\text { Fertilización con boro y NPK. } \\
\text { Manejo y Mantención de plantación: } \\
\text { Control malezas postplantación } \\
\text { Corrección de forma (eliminar daños por helada y polilla) } \\
\text { Poda } 1 \text { (máximo } 30-40 \% \text { de la altura del árbol) } \\
\text { Raleo } 1 \text { a desecho } \\
\text { Poda } 2 \text { (máximo } 20-25 \% \text { copa viva del árbol) } \\
\text { Raleo comercial } \\
\text { Poda final (hasta } 7 \text { m) } \\
\text { Raleo comercial } \\
\text { Cosecha Final } \\
\text { Manejo pradera } \\
\text { Fertilización } \\
\text { Fertilización } \\
\text { Manejo ganadero (ovinos) } \\
\text { Construcción corral } \\
\text { Introducción ganado } \\
\text { Vigilancia ganado } \\
\text { Transporte ganado } \\
\text { Gastos financieros ganado }\end{array}$ \\
\hline
\end{tabular}




\section{- T3: Sistema Silvopastoral (PN 625).}

Pinus radiata con 625 arb.ha $^{-1}$ - pradera natural - ganado ovino.

\section{Cuadro $\mathrm{N}^{\circ} 5$}

ESQUEMA DE MANEJO SISTEMA SILVOPASTORAL DE Pinus radiata CON 625 arb.ha-1 EN CONGLOMERADO CON PRADERA NATURAL Y GANADO OVINO

\begin{tabular}{|c|c|c|c|}
\hline Año & $\begin{array}{l}\text { Densidad } \\
\text { residual } \\
\text { (arb.ha-1) }\end{array}$ & $\begin{array}{c}\text { Arboles a podar } \\
\text { o extraer } \\
\left.\text { (arb.ha' }{ }^{-1}\right)\end{array}$ & Actividad \\
\hline $\begin{array}{c}1 \\
3 \\
5 \\
6 \\
7 \\
8 \\
10 \\
10 \\
24\end{array}$ & $\begin{array}{l}625 \\
625 \\
625 \\
625 \\
318 \\
313 \\
226 \\
226 \\
200\end{array}$ & $\begin{array}{l}468 \\
318 \\
313 \\
226 \\
200 \\
200 \\
200\end{array}$ & $\begin{array}{l}\text { Establecimiento de la plantación: } \\
\text { Habilitación de terreno (roce y ordenamiento desechos) } \\
\text { Preparación del suelo para plantación (subsolado, } \\
\text { camellones curva nivel) } \\
\text { Construcción de cerco, control de malezas preplantación } \\
\text { Plantación densidad } 625 \text { arb.ha-1, en conglomerados de } \\
2 x 2 \text { m y } 6 \text { m entre conglomerados. } \\
\text { Fertilización con boro y NPK. } \\
\text { Manejo y Mantención de plantación: } \\
\text { Control malezas postplantación } \\
\text { Corrección de forma (eliminar daños por helada y polilla) } \\
\text { Poda } 1 \text { (máximo } 30-40 \% \text { de la altura del árbol) } \\
\text { Raleo } 1 \text { a desecho } \\
\text { Poda } 2 \text { (máximo } 20-25 \% \text { copa viva del árbol) } \\
\text { Raleo comercial } \\
\text { Poda final (hasta } 7 \text { m) } \\
\text { Raleo comercial } \\
\text { Cosecha Final } \\
\text { Manejo pradera } \\
\text { Sin manejo } \\
\text { Manejo ganadero (ovinos) } \\
\text { Construcción corral } \\
\text { Introducción ganado } \\
\text { Vigilancia ganado } \\
\text { Transporte ganado } \\
\text { Gastos financieros ganado }\end{array}$ \\
\hline
\end{tabular}




\section{T4: Sistema Forestal (TESTIGO 1600).}

Con Pinus radiata

Este sistema consiste en una plantación forestal, con una rotación de 24 años a contar desde el año 1983. La densidad de plantación fue de 1.600 arb.ha ${ }^{-1}$, a un espaciamiento de $2 \times 3 \mathrm{~m}$. En el siguiente cuadro se detalla el esquema de manejo para la rotación completa del sistema.

\section{Cuadro $\mathrm{N}^{\circ} 6$}

ESQUEMA DE MANEJO SISTEMA FORESTAL PURO DE Pinus radiata

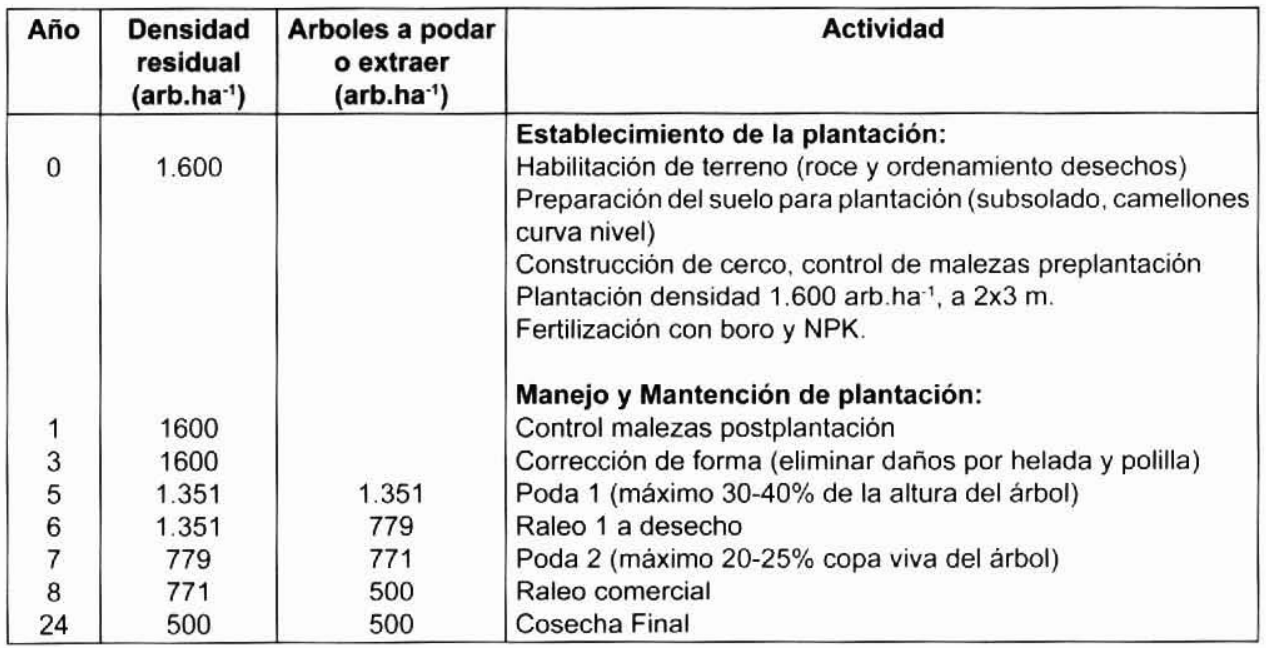




\section{- T5: Sistema pastoral (PS).}

Pradera sembrada - ganado ovino.

Este sistema se estableció el año 1985 con una proyección de 22 años, y corresponde a la siembra de una pradera para producción ovina. La faena de siembra ocurre en el año 0 y la fertilización ocurre durante todos los años. Las faenas relacionadas con la componente ganadera se mantienen por todo el periodo. En el siguiente cuadro se detalla el esquema de manejo para la rotación completa del sistema.

\section{Cuadro $\mathrm{N}^{\circ} 7$}

ESQUEMA DE MANEJO SISTEMA PASTORAL PRADERA SEMBRADA Y GANADO OVINO

\begin{tabular}{|c|l|}
\hline Año & \multicolumn{1}{|c|}{ Actividad } \\
\hline & $\begin{array}{l}\text { Establecimiento y Manejo pradera } \\
\text { Cercado }\end{array}$ \\
$0-5$ & Fertilización \\
$7-15$ & Fertilización \\
$17-22$ & Fertilización \\
& Manejo ganadero (ovinos) \\
1 & Construcción corral \\
1 & Introducción ganado \\
$1-22$ & Vigilancia ganado \\
$1-22$ & Transporte ganado \\
$1-22$ & Gastos financieros ganado \\
\hline
\end{tabular}


T6: Sistema pastoral (PM).

Pradera mejorada - ganado ovino

Este sistema se estableció el año 1986 con una proyección de 21 años, y corresponde a la mantención y mejoramiento de una pradera para ganado. En este caso el mejoramiento de la pradera (fertilización) ocurre en 6 ocasiones durante el periodo. Las faenas relacionadas con la componente ganadera se mantienen por casi todo el periodo. En el siguiente cuadro se detalla el esquema de manejo para la rotación completa del sistema.

\section{Cuadro $\mathrm{N}^{\circ} 8$}

ESQUEMA DE MANEJO SISTEMA PASTORAL, PRADERA MEJORADA Y GANADO OVINO

\begin{tabular}{|c|l|}
\hline Año & \multicolumn{1}{|c|}{ Actividad } \\
\hline & Establecimiento y Manejo pradera \\
$0,3,8,12$ & Cercado \\
15,20 & Fertilización \\
& Fertilización \\
& Manejo ganadero (ovinos) \\
1 & Construcción corral \\
1 & Introducción ganado \\
$1-22$ & Vigilancia ganado \\
$1-22$ & Transporte ganado \\
$1-22$ & Gastos financieros ganado \\
\hline
\end{tabular}

\section{T7: Sistema pastoral (PN).}

Pradera natural - ganado ovino

En este sistema no se incurrió en costos para establecimiento o manejo de la pradera, dado que se trabajo con una pradera natural, y solo se incurrió en costos de manejo ganadero.

\section{Cuadro $\mathrm{N}^{\circ} \mathrm{9}$}

ESQUEMA DE MANEJO SISTEMA PASTORAL, PRADERA NATURAL Y GANADO OVINO

\begin{tabular}{|c|c|}
\hline Año & Actividad \\
\hline $\begin{array}{c}1 \\
1 \\
1-22 \\
1-22 \\
1-22\end{array}$ & $\begin{array}{l}\text { Establecimiento y Manejo pradera } \\
\text { Cercado } \\
\text { Manejo ganadero (ovinos) } \\
\text { Construcción corral } \\
\text { Introducción ganado } \\
\text { Vigilancia ganado } \\
\text { Transporte ganado } \\
\text { Gastos financieros ganado }\end{array}$ \\
\hline
\end{tabular}




\section{Diseño Experimental}

\section{- Numero de parcelas:}

Se instaló tres parcelas por tratamiento, con un diseño al azar, para cada tratamiento con presencia de árboles (T1, T2 y T3), para la evaluación de los parámetros forestales.

\section{Tamaño Parcelas y Variables Respuesta}

Los parámetros a evaluar en los tratamientos con presencia de árboles son:

Diámetro altura del pecho (DAP cm)

Área Basal $\left(\mathrm{AB} \mathrm{m} \mathrm{m}^{2} \cdot \mathrm{ha}^{-1}\right)$,

Altura Total $(\mathrm{H} \mathrm{m})$

Altura Comercial $(\mathrm{H} \mathrm{m})$

Altura de Poda $(\mathrm{m})$

Cobertura de Copa.

T1 a T3: Sistemas Silvopastorales. Parcelas circulares, de $907 \mathrm{~m}^{2}$, distribuidas al azar dentro de las 6 ha del tratamiento, para la evaluación de las variables forestales.

T4: Forestal Puro. Parcelas circulares de $314 \mathrm{~m}^{2}$, distribuidas al azar dentro del área del tratamiento, para la evaluación de las variables forestales.

Los parámetros a evaluar en los tratamientos sin presencia de árboles. Evaluación ganadera y pratense:

Productividad de la Pradera: Para la medición de la productividad de la pradera dentro del tratamiento $\mathrm{T} 4$, en materia seca.ha-1 $(\mathrm{kg})$, se mide las variables de la pradera utilizando jaulas metálicas de exclusión, de una medida de $50 \mathrm{~cm}$ de ancho $\times 100 \mathrm{~cm}$ de largo $\times 50 \mathrm{~cm}$ de alto. Se utilizó jaulas de exclusión para cada tratamiento donde se evalúa la productividad de la pradera de los tratamientos con pradera.

Evaluación Productividad Animal: Para la evaluación de la productividad animal, como incremento en peso vivo $(\mathrm{kg})$, dentro de los tratamiento ganaderos y silvopastorales, se empleó las masas animales de ovinos, de acuerdo a la capacidad de carga.

En el caso de los tratamientos con componente forestal, la evaluación requiere proyectar los resultados a 24 años de rotación. Para esto se emplea el Simulador Radiata para los productos que se detalla en el Cuadro $N^{\circ} 10$. 


\section{Cuadro $\mathrm{N}^{\circ} 10$ \\ DESCRIPCIÓN DE PRODUCTOS UTILIZADOS EN LA SIMULACIÓN \\ Y PRECIOS DE PRODUCTOS POR $\mathrm{m}^{3}$}

\begin{tabular}{|c|c|c|c|c|c|}
\hline PRODUCTO & CALIDAD & $\begin{array}{c}\text { Largo } \\
(\mathbf{m})\end{array}$ & $\begin{array}{c}\text { Diam min } \\
\text { (cm) }\end{array}$ & $\begin{array}{c}\text { Precio (UFI } \\
\mathbf{m}^{3} \text { ) } \\
\end{array}$ & Precio (US/m³) \\
\hline P1 & Podado & 2,80 & 36 & 1,77 & 60 \\
\hline $\mathbf{P 2}$ & Podado & 2,80 & 32 & 1,62 & 55 \\
\hline P3 & Aserrable s/poda & 3,25 & 32 & 1.48 & 50 \\
\hline P4 & Aserrable s/poda & 3,25 & 24 & 1,33 & 45 \\
\hline P5 & Aserrable s/poda & 3,25 & 18 & 1,03 & 35 \\
\hline P6 & Aserrable s/poda & 3,25 & 14 & 0,89 & 30 \\
\hline P7 & Pulpable & 2,44 & 10 & 0,59 & 20 \\
\hline P8 & Leña & 2,44 & 6 & 0,3 & 10 \\
\hline
\end{tabular}

Valores puesto planta

\section{RESULTADOS Y DISCUSIÓN}

\section{Rendimientos Forestales}

Los resultados de la evaluación de cada uno de los factores productivos son expuestos a continuación.

Como se indica en la Figura $\mathrm{N}^{\circ} 1$, la evolución de los parámetros forestales al momento de la cosecha, es decir a los 24 años, siguen los principios tradicionales de una plantación establecida a bajas densidades versus a una a alta densidad.

DAP: Los mayores diámetros se obtienen en los sistemas silvopastorales con diámetros entre los 50 a $52 \mathrm{~cm}$, versus $41 \mathrm{~cm}$ del sistema forestal.

$A B$ : El área basal, que es fuertemente influenciada por la densidad, obtuvo los mayores valores con el tratamiento forestal, seguida por T1 que, dentro de los sistemas silvopastorales, fue el que obtuvo la mayor densidad final con 224 arb. ha $^{-1}$

$\mathrm{H}$ total: La altura total no es influenciada por la densidad, lo cual indica que los tratamientos fueron instalados en una misma calidad de sitio. 


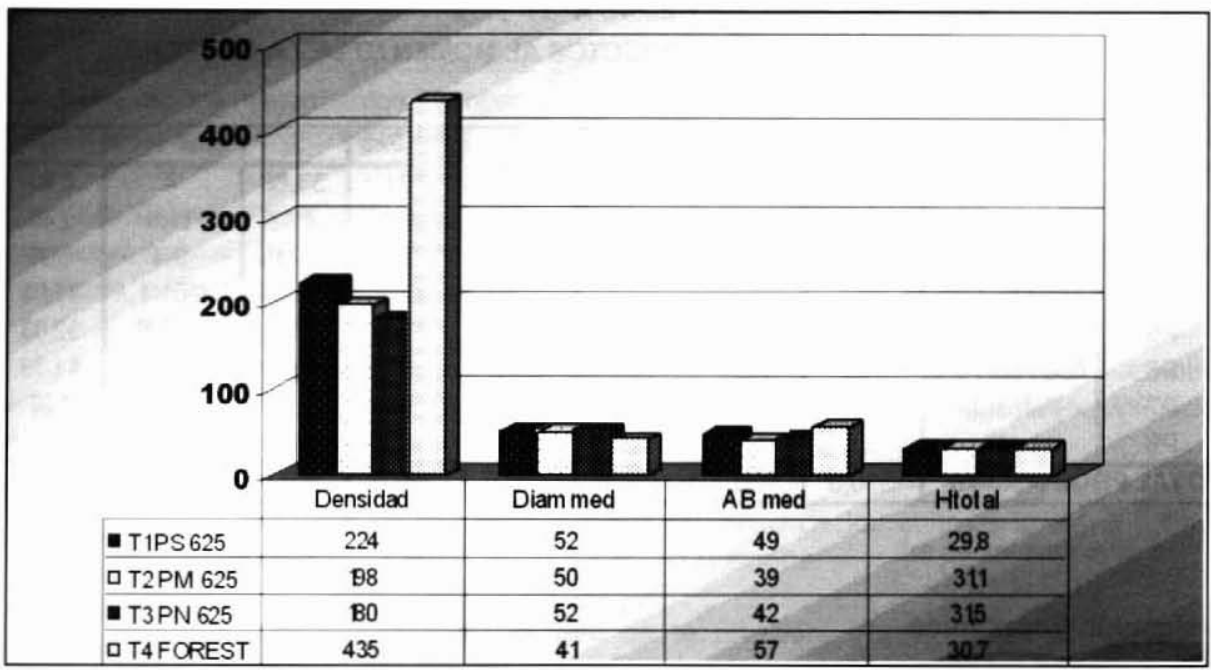

\section{FIGURA $N^{\circ} 1$ \\ RESULTADOS PARÁMETROS FORESTALES A LOS 24 AÑOS}

La calidad de los productos que se obtiene de un manejo forestal está directamente relacionada con el material genético utilizado, la calidad del sitio y el manejo que se da a la plantación. En este caso el material genético y calidad de plantas fue el mismo y los tratamientos fueron instalados en el mismo sitio, de modo que las diferencias en los resultados radican en el manejo y el diseño utilizado.

La mayor productividad en términos de volumen total se obtuvo en el tratamiento forestal, con $479.5 \mathrm{~m}^{3}$.ha ${ }^{1}$, versus una productividad similar entre los tratamientos silvopastorales (Cuadro $\mathrm{N}^{0} 11$ ). La diferencia se produce en cuanto a la calidad de los productos obtenidos y a la distribución de estos productos en la producción total. Los productos podados, que son los que tienen un mayor precio, entregan entre un 37 a $38 \%$ del volumen total en los tratamientos silvopastorales, versus solo un $3,1 \%$ en el sistema forestal. En el sistema forestal el volumen mayor se concentra en P4 y P5, que son productos intermedios en cuanto al valor, lo cual incidirá en la rentabilidad del sistema. 


\section{Cuadro $\mathrm{N}^{\circ} 11$}

PRODUCCIÓN POR TIPO DE PRODUCTOS AL MOMENTO DE LA COSECHA

\begin{tabular}{|c|c|c|c|c|c|c|c|c|c|}
\hline Producto & Calidad & PS 625 & $\%$ & PM625 & $\%$ & PN 625 & $\%$ & Test 1600 & $\%$ \\
\hline P1 & Podado & 120,7 & 30,95 & 122,2 & 31,58 & 131 & 33,68 & 4 & 0,83 \\
P2 & Podado & 27,2 & 6,97 & 25,1 & 6,49 & 17 & 4,37 & 11,5 & 2,40 \\
P3 & Aserrable s/p & 82,2 & 21,08 & 85,5 & 22,10 & 95 & 24,43 & 9,4 & 1,96 \\
P4 & Aserrable s/p & 95,1 & 24,38 & 87,7 & 22,67 & 87,3 & 22,45 & 201,1 & 41,94 \\
P5 & Aserrable s/p & 37,6 & 9,64 & 37,8 & 9,77 & 32,5 & 8,36 & 153,6 & 32,03 \\
P6 & Aserrable s/p & 13,2 & 3,38 & 15,1 & 3.90 & 14 & 3,60 & 54,6 & 11,39 \\
P7 & Pulpable & 10,2 & 2,62 & 9,9 & 2,56 & 8,8 & 2,26 & 33,4 & 6,97 \\
P8 & Leña & 3,8 & 0,97 & 3,6 & 0,93 & 3,3 & 0,85 & 11,9 & 2,48 \\
\hline
\end{tabular}

Volúmenes en $\mathrm{m}^{3}$

\section{Evaluación Económica}

La evaluación económica de los tratamientos comparados toma en cuenta todos los ingresos, forestales y animales, como los incentivos que entrega el Gobierno en base a subsidios a la forestación y a la recuperación de suelos, durante toda la rotación de los tratamientos. También toma en cuenta los costos de cada uno de los tratamientos evaluados y expuestos en el punto descripción de tratamientos.

Los resultados que se expone en el Cuadro $\mathrm{N}^{\circ} 12$, permiten notar que se obtiene los mejores resultados en aquellos tratamientos donde participa la componente forestal. Los mejores resultados en cuanto a VAN y TIR se encuentran en los dos tratamientos silvopastorales, T2 y T3, y en el tratamiento forestal T4, sin diferencias significativas entre ellos. Las menores rentabilidades están en los tratamientos ganaderos con ovinos y, en especial, en aquellos donde participa la componente pradera sembrada, lo cual es similar en el caso silvopastoral. Esto se debe al alto costo de la fertilización que una pradera sembrada con trébol subterráneo y falaris requiere para su desarrollo.

\section{Cuadro $\mathrm{N}^{\circ} 12$ \\ EVALUACIÓN ECONÓMICA DE LOS TRATAMIENTOS COMPARADOS CON Y SIN BONIFICACIÓN FORESTAL}

\begin{tabular}{|l|c|c|c|c|}
\cline { 2 - 5 } \multicolumn{1}{c|}{} & \multicolumn{2}{c|}{ CON BONIFICACION } & \multicolumn{2}{c|}{ SIN BONIFICACION } \\
\hline TRATAMIENTOS PRODUCTIVOS & VAN (10\%) & TIR (\%) & VAN (10\%) & TIR (\%) \\
\hline T1, Silvopastoral 625-Pradera sembrada & 73,75 & $12,40 \%$ & $-10,82$ & $9,70 \%$ \\
\hline T2, Silvopastoral 625-Pradera mejorada & 148,38 & $16.20 \%$ & 52,81 & $11,50 \%$ \\
\hline T3, Silvopastoral 625- Pradera natural & 141,94 & $16,16 \%$ & 44,33 & $11,28 \%$ \\
\hline T4, Testigo Forestal 1600 & 108,34 & $15,50 \%$ & 3,44 & $10,10 \%$ \\
\hline T5, Pradera sembrada & $-180,59$ & Indet & $-188,73$ & Indet \\
\hline T6, Pradera mejorada & $-49,26$ & Indet & $-57,55$ & Indet \\
\hline T7, Pradera natural & 0,84 & 10,45 & 0,84 & $10,45 \%$ \\
\hline
\end{tabular}




\section{CONCLUSIONES}

Los resultados entregados en este estudio, arrojan interesantes cifras en la evaluación económica de los tratamientos comparados. Los mejores resultados se obtienen cuando participa la componente forestal, es decir los sistemas silvopastorales y el forestal.

Los sistemas silvopastorales con pradera natural manejada (TIR 16,2\%), silvopastoral con pradera natural (TIR 16,0\%), y forestal puro (TIR 15,5\%) ofrecen los mejores resultados Por el contrario, en todos los sistemas ganaderos las rentabilidades fueron negativas.

Los resultados indican que los sistemas silvopastorales son una interesante alternativa para los pequeños propietarios de suelos de secano, dado que son los sistemas mejor evaluados en cuanto a rentabilidad y además ofrecen beneficios como:

Obtención de ingresos anuales producto de los cultivos anuales y de la producción animal, en este caso con ovinos.

Interesante ingreso al final de la rotación, por la venta de productos forestales de una alta calidad originada en el manejo de los bosques.

- Sienta una base para fomentar la forestación con fines diferentes a los tradicionales en la legislación de fomento gubernamental, lo que resultaría de gran valor e interés para los pequeños propietarios, dado que les permitiría mantener sus sistemas productivos, y adaptarlos más fácilmente con un manejo forestal.

- Mantener a los propietarios en sus tierras, evitando la migración a las ciudades, lo cual los desarraiga, origina pobreza marginal y cambia su sistema cultural agricola.

En los sistemas silvopastorales, al realizar un manejo forestal intensivo con fines de producción de madera libre de nudos y de alta calidad, arroja interesantes resultados económicos debido a la obtención de productos podados, que influencian positivamente los resultados de la evaluación económica.

\section{REFERENCIAS}

Anderson, G.W., Moore, R.W. and Jenkins, P.J., 1988. The integration of pasture, livestock and widelyspaced pine in South West Western Australia. Agroforestry Systems, 6: 195-211.

Leslie, B., Knowles, R.y Moore, R, 1998. Silvopastoreo con Pinus radiata en Zonas Frias. In: Compilación de Resultados en Diversos Ensayos de Modelos Silvopastorales en Chile y en el Extranjero.

Polla, C., 1998. Estrategias de Acción en el Tema Silvopastoreo. IN: Actas Seminario "Manejo Silvopastoral" Trabajo $\mathrm{N}^{\circ} 8$, Young. Uruguay. 
Quam, V. and Johnson, I., 1999. Windbreaks for Livestock Operations. University of Nebraska Cooperative Extension EC 94-1766-X. Recuperado el 31 de Enero de 2002 de World Wide Web: http:/l www. Ianr.unl edu/pubs/forestry/ec1766.htm

Rodriguez, M., 1998. Evaluación Económica del Sistema Silvopastoral Pino/Oveja Presente en el Centro Experimental Forestal Tanume CONAF, VI Región. Memoria Facultad de Ciencias Agrarias y Forestales, Universidad de Chile. Santiago, Chile

Sotomayor, A., 1989. Sistemas Silvopastorales y su Manejo. Chile Agricola, (156):203-206.

Sotomayor, A., 1990. Sistemas Silvopastorales y su Manejo. Chile Agrícola, (157):203-206.

Sotomayor, A., Garcia E. 2004. Cartilla Agroforestal N² 2, Sistemas Silvopastorales, INFOR, 2004.

www.agroforesteria.cl, 2007. Red Agroforestal Nacional, Publicaciones, Cartillas Agroforestales, INFOR, Concepcion, Chile. 